\title{
THE OPTIMIZATION OF DIFFERENTIAL REFLECTOMETRY AT SURFACES OF TRANSPARENT CRYSTALS
}

\author{
L. WOLTERBEEK, G.F. AKKERHUIS and A. VAN SILFHOUT \\ Department of Applied Physics, Twente University of Technology, P.O. Box 217, 7500 AE Enschede, \\ The Netherlands
}

Received 1 April 1984; accepted for publication 12 June 1984

The first-order difference in reflectivity of crystals surfaces due to the adsorption of a (sub)monolayer is calculated for crystals transparent to the incident radiation. Conditions are derived for optimizing experimental studies of both strongly and weakly absorbing surfaces and/or adsorbates. The large and small absorption bandwidths involved correspond respectively to (i) electronic transitions to/from surface, interface and adsorbate states, and, (ii) vibrational excitations of adsorbates. The variable parameters are the angle of the incident light beam and the state of the back surface. It is found that the sensitivity varies with angle of incidence, depending on the type of absorbing monolayer.

\section{Introduction}

Optical methods are widely used to study the physical or chemical state of crystal surfaces. Slight variations of the state of a surface induce measurable alterations in the specular reflectivity, such as the phases or the intensities of the reflected beam with polarization parallel (p) or perpendicular $(s)$ to the plane of incidence.

If the crystal is transparent to the incident beam, e.g. silicon with infrared light having photon energy below the indirect band gap of $1.1 \mathrm{eV}$, the back surface of the crystal and a reflecting object behind the crystal will also contribute to the total reflectivity. If the wavelength bandwidth is not very narrow or the surfaces not completcly planc-parallel, measurements based on a phase relation between incident and emergent beam (ellipsometry) become questionable and only the possibility of intensity measurement remains.

For perpendicularly incident light the reflection by the front and back surface of a crystal has been treated by del Sole [1]. He considered the influence of varying the front and/or the back surface condition of a planeparallel crystal both on the reflected and the transmitted beam.

In practice, however, a crystal is mounted in front of a crystal holder for positioning and temperature control. In this way transmission measurements 
are very difficult to perform. On the other hand it is possible to realize a flat metallic surface with known optical constants, behind the crystal, thus influencing the reflectivity in a predictable way. Angular dependent reflection measurements in this manner ar thus possible.

\section{Angular variation of the total reflectivity}

Suppose that the transparent crystal slab is illuminated over its front surface at different angles of incidence. Variations in the reflectivity caused by differences in the infrared ( $2-10 \mu \mathrm{m}$ wavelength) surface response correspond to the presence of realistic states at the silicon surface in the monolayer region.

These states are: surface electron states optically active at about $2 \mu \mathrm{m} \mathrm{[2],}$ $\mathrm{Si}-\mathrm{H}$ vibrations $[3,4]$ around $5 \mu \mathrm{m}$, and $\mathrm{Si}-\mathrm{N}$ and $\mathrm{Si}-\mathrm{O}-\mathrm{Si}$ stretch vibrations around $10 \mu \mathrm{m}$. At these wavelengths the influence of the deposition of monolayers of metals (platinum and molybdenum) is also studied.

The calculation of the reflectivity is based on the assumption of complete incoherence between light travelling back and forth between the surfaces, due to the assumed bandwidth of $\sim 20 \mathrm{~cm}^{-1}$, and the surfaces being of the order of a millimeter apart.

The calculation of the resulting total reflection, $R_{\text {total }}$, proceeds as follows (see fig. 1).

The radiation incident through vacuum (medium 1) induces a light flux $\Phi_{24}$ through the substrate from the front surface, clean or covered with an absorbing film, 2 to the back surface, also clean or covered with a layer 4 . In the opposite direction, within the crystal, flux $\Phi_{42}$ travels from back (4) to front (2) surface. The space between the back face 4 of the crystal and the metal mirror 5 is crossed by the fluxes $\Phi_{45}$ and $\Phi_{54}$ respectively.

When $T_{i(j) k}$ and $R_{i(j) k}$ are defined as the transmission and reflection factors, respectively, at the transition of medium $i$ to medium $k$, with or without absorbing film $j$ inbetween, then the following balance equations exist for the light fluxes, the incident flux taken to be unity:

$\Phi_{24}=T_{1(2) 3}+\Phi_{42} R_{3(2) 1}$,

$\Phi_{42}=\Phi_{24} R_{3(4) 1}+\Phi_{54} T_{1(4) 3}$,

$\Phi_{45}=\Phi_{24} T_{3(4) 1}+\Phi_{54} R_{1(4) 3}$,

$\Phi_{54}=\Phi_{45} R_{15}$,

$R_{\text {total }}=R_{1(2) 3}+\Phi_{42} T_{3(2) 1}$.

With the aid of eqs. (1)-(4), the flux $\Phi_{42}$ can be expressed in terms of the constants $R$ and $T$ (and the unity incident flux), and substituted in eq. (5). 


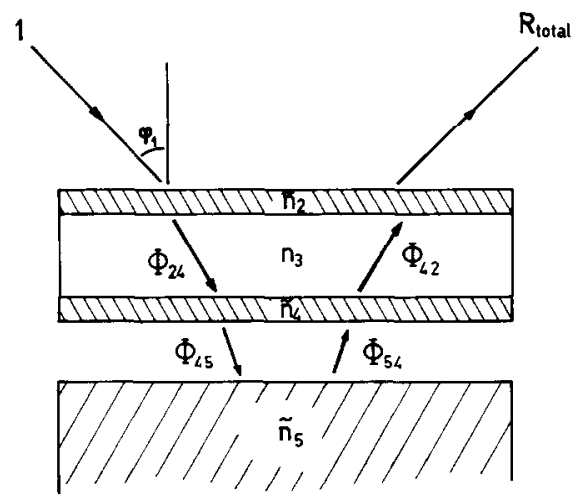

Fig. 1. Light fluxes inside and outside a transparent crystal (real refraction index $n_{3}$ ). The front and back surfaces may be covered with absorbing monolayers with complex refraction indices $\tilde{n}_{2}$ and $\tilde{n}_{4}$, respectively.

Straightforward calculation gives:

$$
\begin{aligned}
R_{\text {total }}= & R_{1(2) 3}+T_{3(2) 1} T_{1(2) 3}\left(R_{3(4) 1}+\frac{T_{3(4) 1} R_{15} T_{1(4) 3}}{1-R_{15} R_{1(4) 3}}\right) \\
& \times\left(1-R_{3(2) 1} R_{3(4) 1}-\frac{R_{3(2) 1} T_{3(4) 1} R_{15} T_{1(4) 3}}{1-R_{15} R_{1(4) 3}}\right)^{-1} .
\end{aligned}
$$

Further, for knowledge of $R_{\text {total }}$ both in the presence and absence of the absorbing films $j$ we need to know the reflection and transmission factors $R_{i(j) k}$ and $T_{i(j) k}$. For the clean surfaces $R_{i k}$ end $T_{i k}$ are simply given by the Fresnel coefficients and are not repeated here [5]. The reflection factors $R_{i j k}$ with intermediate absorbing film $j$ (index $\tilde{n}_{j}=n_{j}-\mathrm{i} k_{j}$ ) are expressed in the reflection factors $R_{i k}$ of the clean surface $k$ by means of the calculation to the first order of the layer thickness $d_{j}\left(d_{j} \ll \lambda\right.$, the vacuum wavelength) of McIntyre and Aspnes [6]:

perpendicular polarization, $s$ :

$$
\left(\frac{R_{i j k}}{R_{i k}}\right)_{\mathrm{s}}=1+\frac{16 \pi d_{j} n_{i} \cos \phi_{i} n_{j} k_{j}}{\lambda\left(n_{k}^{2}-n_{i}^{2}\right)},
$$

parallel polarization, $\mathbf{p}$ :

$$
\begin{aligned}
\left(\frac{R_{i j k}}{R_{i k}}\right)_{\mathrm{p}}= & 1+16 \pi d_{j} n_{i} \cos \varphi_{i} n_{j} k_{j}\left\{\lambda\left(\frac{n_{k}^{2}}{n_{i}^{2}}-1\right)\left[1-\left(1+\frac{n_{i}^{2}}{n_{k}^{2}}\right) \sin ^{2} \varphi_{i}\right]\right\}^{-1} \\
& \times\left\{\frac{1}{n_{i}^{2}}-\left[\frac{1}{n_{k}^{2}}+\frac{2 n_{k}^{2}}{\left(n_{k}^{2}+k_{j}^{2}\right)^{2}}\right] \sin ^{2} \varphi_{i}\right\} .
\end{aligned}
$$




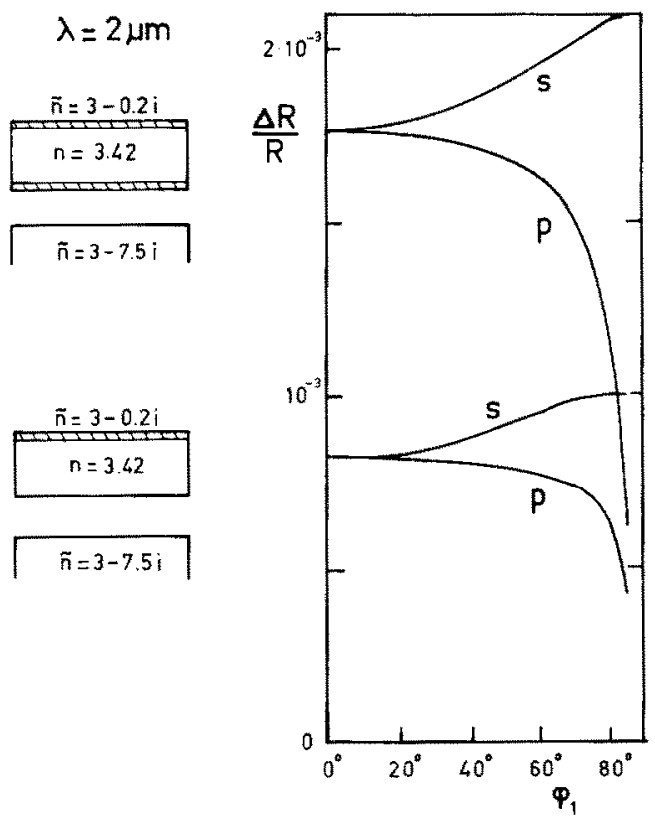

Fig. $2 . \lambda=2 \mu \mathrm{m}$, Si(111) $-2 \times 1$ surface with $-10^{12}$ surface states $/ \mathrm{cm}^{2}$.
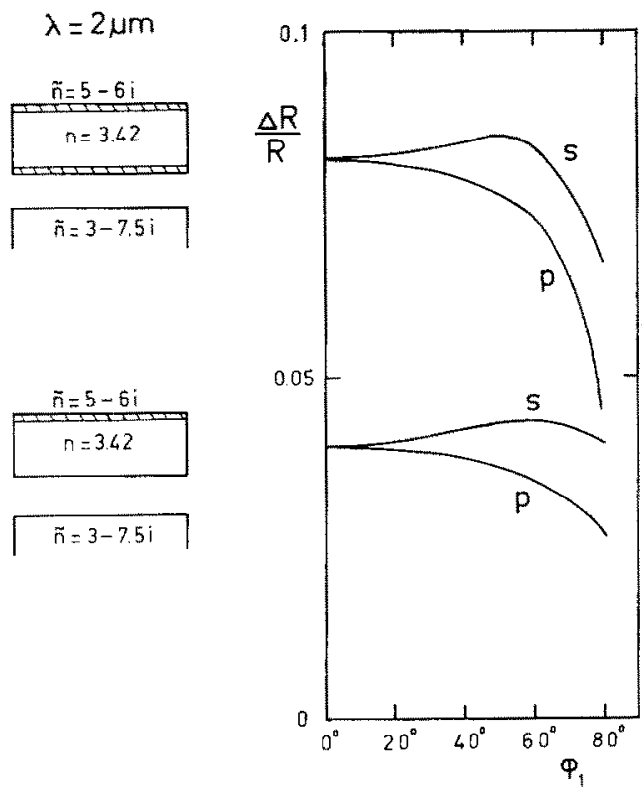

Fig. 3. $\lambda=2 \mu \mathrm{m}$, Si surface with monolayer of Mo. 
Here $\varphi_{i}$ is the angle of the direction of progation in medium $i$, relative to the surface normal. In a similar manner to that applied in ref. [6] one can derive, to first order, for the ratio of the transmission coefficients:

$$
\begin{aligned}
& \left(\frac{T_{i j k}}{T_{i k}}\right)_{\mathrm{s}}=1-\frac{8 \pi d_{j} n_{j} k_{j}}{\lambda\left(n_{i} \cos \varphi_{i}+n_{k} \cos \varphi_{k}\right)}, \\
& \left(\frac{T_{i j k}}{T_{i k}}\right)_{\mathrm{p}}=1-8 \pi d_{j} n_{j} k_{j}\left[\lambda\left(\frac{\cos \varphi_{i}}{n_{i}}+\frac{\cos \varphi_{k}}{n_{k}}\right)\right]^{-1}\left(\frac{\cos \varphi_{i} \cos \varphi_{k}}{n_{i} n_{k}}+\frac{n_{i}^{2} \sin \varphi_{i}}{n_{j}^{2}+k_{j}^{2}}\right) .
\end{aligned}
$$

From Snell's law it follows, that

$\sin \varphi_{i}=\frac{\sin \varphi_{1}}{n_{i}}, \quad \cos \varphi_{i}=\left(1-\frac{\sin ^{2} \varphi_{1}}{n_{i}^{2}}\right)^{1 / 2}$,

$\varphi_{1}$ being the angle of incidence of the beam from vacuum.

\section{Angular variation of the differential reflectivity}

By means of eq. (6) the reflectivity of a silicon crystal is determined with and without the absorbing layers 2 and 4, from which the relative reflection

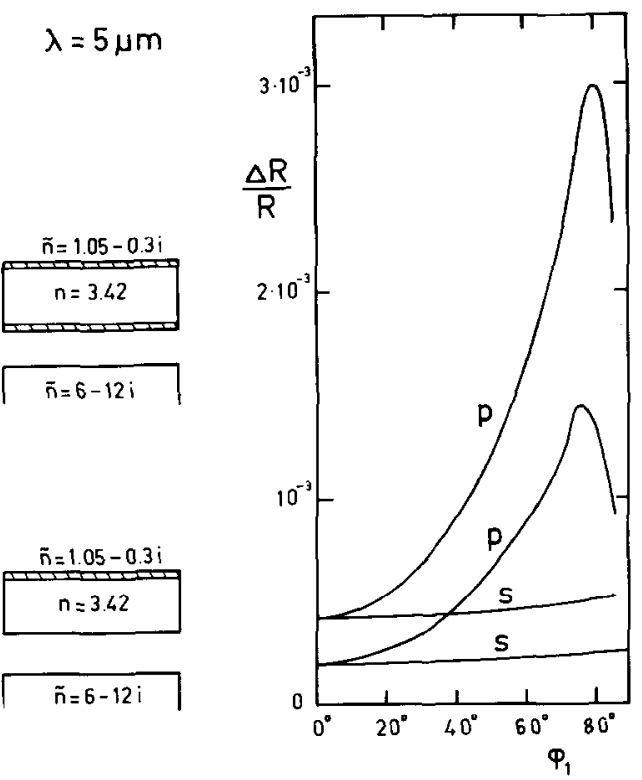

Fig. 4. $\lambda=5 \mu \mathrm{m}, \mathrm{Si}$ surface with monolayer of $\mathrm{Si}-\mathrm{H}$. 


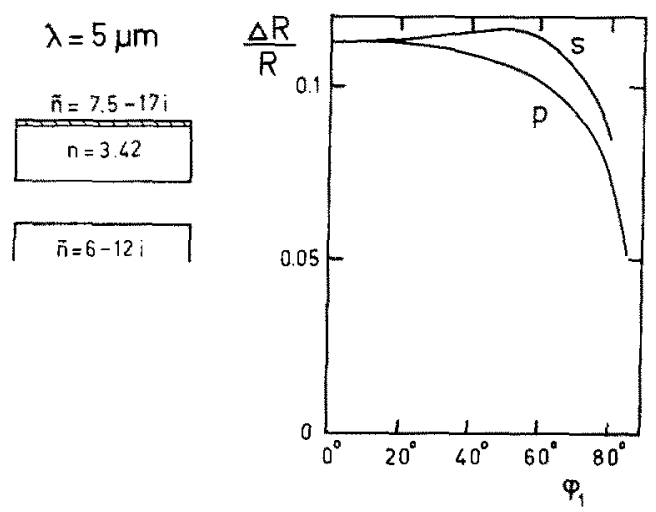

Fig. S. $\lambda=5 \mu \mathrm{m}$, Si surface with monolayer of $\mathrm{Pt}$.

change has been calculated. This is defined here, to obtain positive numbers, as:

$$
\frac{\Delta R}{R}=\frac{R_{\text {total }}(\text { clean })-R_{\text {total }}(\text { with layers })}{R_{\text {total }}(\text { clean })} .
$$

The designation "clean" refers to the absence of a surface layer which would absorb in the wavelength region of interest.

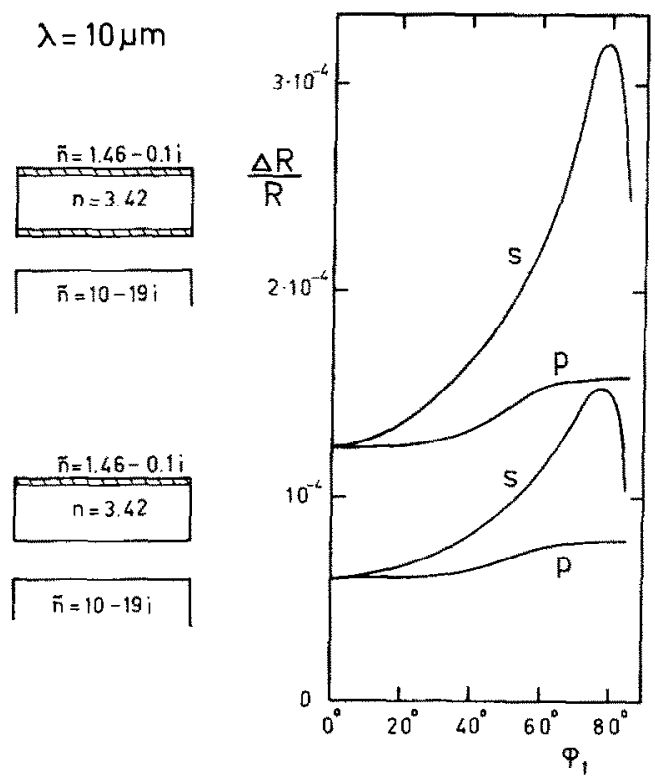

Fig. 6. $\lambda=10 \mu \mathrm{m}, \mathrm{Si}$ surface with monolayer of $\mathrm{SiO}_{x}$. 


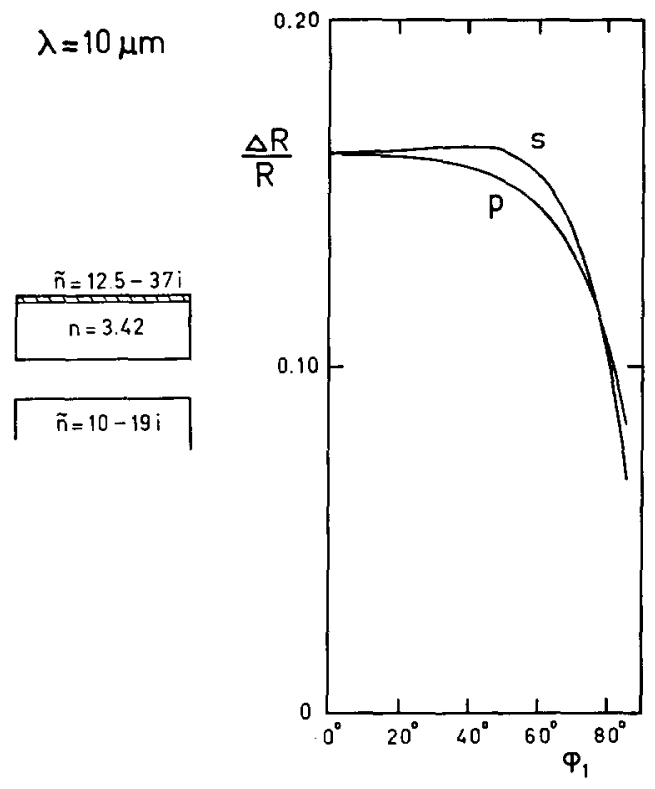

Fig. 7. $\lambda=10 \mu \mathrm{m}$, Si surface with monolayer of Pt.

In figs. 2-7 the results are exhibited, showing the relative reflectivity change for $s$ and $p$ polarized light, as a function of the angle of incidence $\varphi_{1}$. It is supposed, that over the entire range of angles of incidence the full beam width is contained within the sample dimensions. The backing mirror is assumed to be tantalum because of its applicability in ultra-high vacuum systems in combination with its fair reflectivity in the infrared $[7,8]$.

\section{Conclusions}

From the graphs the following conclusions can be drawn:

(a) Weakly absorbing states having a small value for the real part of the refraction index are most sensitive to parallel polarized (p) reflection at large angles of incidence $\varphi_{1}$, similar to absorbing states on metal surfaces [9], although in this case the effect is far less pronounced.

(b) Weakly or moderately absorbing states having a real part of the refraction index roughly equal to the substrate index show the opposite effect: the sensitivity, with respect to $\mathrm{p}$ polarization, sharply increases with $\varphi_{1}$. Whereas $(\Delta R / R)_{\mathrm{s}}$ remains almost constant, $(\Delta R / R)_{\mathrm{p}}$ decreases by a factor of about 5 at $\phi_{1}=80^{\circ}$.

(c) For metallic surface states, $(\Delta R / R)_{\mathrm{s}}$ and $(\Delta R / R)_{\mathrm{p}}$ are roughly equal and both decrease slowly with $\varphi_{1}$. 
A direct comparison of these results with experiment is not possible since measurements based on the above system have not yet been performed. Thus far infrared reflection at monolayers on semiconductor crystals has only been studied by means of internal reflection [2,4]. This enhances the differential effect by the multiplicity $\left(\approx 10^{2}\right)$ of reflection, at the cost, however, of reducing both the optical étendue (beam area times solid angle) and the wavelength region of transmission through silicon. The detection limit is $\Delta R / R \approx 10^{-4}$ per reflection.

On the other hand, external $\Delta R / R$ measurements in the infrared have only been carried out at monolayers on metals, for which the detection limit is $\Delta R / R \approx 10^{-3}$. Due to the "infrared selection rule" only $\mathrm{p}$ reflection can be applied, at grazing incidence [9]. Under these conditions $R_{\mathrm{p}}$ and $R_{\mathrm{v}}$ are both approximately unity: the technique of polarization modulation can therefore be applied leading to a detection limit of $2 \times 10^{-4}$ in single beam operation [10]. This latter technique may not easily be extended to the present case since the angle of incidence differs from grazing.

From the above it would appear that, even for cases of low differential reflection (e.g. fig. 4: $\Delta R / R=3 \times 10^{-3}$ at $\varphi_{1}=80^{\circ}$ ), complete monolayer detection is possible.

A comparison of this work (external reflection) with internal reflection will be dealt with in a forthcoming publication.

\section{References}

[1] R. del Sole, Surface Sci. 123 (1982) 231.

[2] G. Chiarotti, S. Nannarone, R. Pastore and P. Chiaradia, Phys. Rev. B4 (1971) 3398.

[3] V.J. Kapoor, R.S. Bailey and H.J. Stein, J. Vacuum Sci. Technol. A1 (1983) 600.

[4] Y.J. Chabal, E.E. Chaban and S.B. Christman, J. Electron Spectrosc. Related Phenomena 29 (1983) 35.

[5] M. Born and E. Wolf, Principles of Optics (Pergamon, 1970) p. 40.

[6] J.D.E. McIntyre and D.E. Aspnes, Surface Sci. 24 (1971) 417.

[7] American Institute of Physics Handbook (McGraw-Hill, New York, 1972).

[8] A.P. Lenham and D.M. Treherne, J. Opt. Soc. Am. 56 (1966) 1137.

[9] R.G. Greenler. J. Vacuum Sci. Technol. 12 (1975) 1410.

[10] W.G. Golden, D.S. Dunn and J. Overend, J. Catalysis 71 (1981) 395. 